\title{
The Electromotive Force Dependence on the Polycrystalline Silicon Solar Cell Illuminance
}

\author{
A. Jukna, P. Miškinis, V. Valuntaité And A. Bogdanovičius \\ Radiation Research Laboratory, Department of Physics, Faculty of Fundamental Sciences, Vilnius Gediminas \\ Technical University, Sauletekio Ave.11, LT-10223, Vilnius, Lithuania
}

\begin{abstract}
The impact of illuminance on changes of the solar cell electromotive force is analyzed. A mathematical model for a solar cell electromotive force dependence on illuminance is presented. For this purpose, a selection of experimental data trend function was carried out, and the Pearson correlation coefficients were established. The most optimal results were obtained in case of an exponential function with the strongest correlation $\left(R^{2}=0.983\right)$. The analysis has shown that at $100 \mathrm{~W} / \mathrm{m}^{2}$ illuminance the electromotive force saturation is obtained (the electromotive force changes insignificantly and fluctuates at around $2 \mathrm{~V}$ ), which indicates that upon reaching such an illuminance a solar cell operates at maximum efficiency. A first-order differential equation satisfied by the trend function has been compiled. When interpreting illuminance as an evolution variable, the proposed mathematical model can be interpreted as a dynamical system. The deviation frequency spectrum of the measurement values with respect to the theoretical prediction is analyzed.
\end{abstract}

DOI: 10.12693 /APhysPolA.127.1711

PACS: 88.40.H-, 88.40.jj, 88.40.hj, 88.40.fc

\section{Introduction}

As oil supplies are decreasing and the global warming is threatening, photovoltaic devices are becoming more and more popular as a renewable and environmentally friendly energy alternative [1, 2]. Solar cells are electronic devices which turn sunlight into electricity $[3,4]$. A significant advantage of solar cells, as compared with ordinary fuel, is their ability to turn the free radiation obtained from the sun into electricity with almost no pollution emissions. Solar cells can be classified into three main categories: monocrystalline silicon, polycrystalline silicon, and amorphous silicon. The efficiency of monocrystalline silicon solar cells is the highest $(25 \%)$ as compared with polycrystalline silicon (20\%) and amorphous silicon (10\%) solar cells, but they are most expensive. Crystalline silicon solar cells are used most often (around $90 \%$ ), and their coefficient of performance reaches 16$17 \%$. The manufacturing and usage of multijunction thinfilm solar cells, however, is gradually increasing (the coefficient of their performance reaches $55 \%)[5,6]$.

The applications are truly endless [7] — from solar thermal decoupled water electrolysis process $[8]$ and the solar thermal electrolytic production of $\mathrm{Mg}$ from $\mathrm{MgO}$ [9] up to using solar energy for cooling in the agro-food industries (e.g. [10]).

When looking for solutions to decrease the increasing energy prices and to reduce the environmental impact caused by energy, an increasingly bigger role is played by renewable energy sources, one of which is solar energy [11]. The option of solar energy usage is a solution for many people who have no access to an electricity network due to distance, small number of inhabitants, poverty or geographical situation. In the recent years, solar energy usage has increased because of improved technologies, reduced spending on production, and the governmental policy encouraging the renewable energy development $[12,13]$. Solar energy is being widely used worldwide as a source of energy for individual houses, commercial buildings, in industry, as well as for the lighting of streets, gardens and parks, and for water pumps.

Although the Lithuanian geographical latitude is not very favourable for solar energy usage as compared with that of the countries located closer to the Equator, the solar energy falling onto the earth surface here diffuses on a much more larger surface than in the geographical latitudes where the sun is in zenith at midday. The annual amount of solar energy falling onto the surface of $1 \mathrm{~m}^{2}$ area in Lithuania exceeds $1000 \mathrm{kWh}$ [14]. In the Lithuanian climate conditions, the cost of installation of $1 \mathrm{~kW}$ solar energy production reaches EUR 2.9-4.1 thousand per year. A photo-voltage system with a $1 \mathrm{~kW}$ installed capacity produces $880-940 \mathrm{kWh}$ of electricity, and the cost of its production is $0.41-0.43 \mathrm{EUR} / \mathrm{kWh}$ [15]. Photoelectricity is currently rather expensive, but with the rapid development of technologies the prices are forecasted to become equal in 2018-2020. Lithuania has an obligation to the European Union to increase the share of renewable energy sources (RES) in electricity production to at least $20 \%$ (which also include $10 \mathrm{MW}$ of solar power plants) until 2020. Currently, the energy obtained from RES makes around $15 \%$ of the final energy consumption in Lithuania [16]. Thus, the influence of illuminance on the total efficiency of the solar cell is an important problem of their functioning. On the other hand, the solar cell electromotive force (EMF) is one of the basic characteristics of its operation.

The aim of the study was to examine changes of a solar cell EMF, the reasons determining changes under various conditions for illuminance, and to draw a mathematical model for a solar cell EMF dependence on illuminance. 


\section{The characteristic equation of a solar cell}

An ideal solar cell may be modelled by a current source in the circuit with a parallel diode [17]. In practice, no solar cell is ideal, so a shunt resistance $R_{\mathrm{sh}}$ and a series resistance component $R_{\mathrm{S}}$ are added to the model [1820]. The resulting equivalent circuit of a solar cell is shown in Fig. 1.

The characteristic equation of a solar cell, which relates solar cell parameters to the output current $I$ and voltage across the output terminals $U[17,20]$, is

$$
\begin{aligned}
I & =I_{L}-I_{0}\left[\exp \left(\frac{q\left(U+I R_{\mathrm{s}}\right)}{\alpha k T}\right)-1\right]- \\
& -\frac{q\left(U+I R_{\mathrm{s}}\right)}{R_{\mathrm{sh}}},
\end{aligned}
$$

where $I_{L}$ is the photo-generated current, $I_{0}$ is the reverse saturation current, $q$ is the elementary charge, $\alpha$ is a diode ideality factor $(1<\alpha \leq 2)$ (in practice, at low voltages $\alpha \rightarrow 2$, whereas at high voltages $\alpha \rightarrow 1$, see, e.g., $[19,21]), k$ is the Boltzmann constant, and $T$ is the absolute temperature. Because the equation involves the output current $I$ on both sides in a transcendental function, the equation has no general analytical solution, but it is easily solved by numerical methods. A more detailed situation is the following: a general analytical solution of the characteristic equation is possible using the Lambert $W(x)$ or the product-log function, but since the Lambert $W$-function itself generally must be solved numerically, this analiticity disappears.

Since the parameters $I_{0}, \alpha, R_{\mathrm{s}}$, and $R_{\mathrm{sh}}$ cannot be measured directly, the most common application of the characteristic equation is nonlinear regression to extract the values of these parameters on the basis of their combined effect on solar cell behavior.

Taking into account the range of illuminance of our tested solar cells, we do not consider the exceptional behavior of light-matter interaction in the extreme ultraviolet and possible deviations of the classical external photoeffect (see, e.g., [22]).

\section{Collection of experimental data}

A fixed exposition stand with two solar cells connected through an analog-digital converter to the computer was used in our experiments. The experiments were done during a daytime in the period from March 18 to May 13 2014. Two solar cells (SCs) were installed on a shadowfree roof-top plane, whereas a computer and a data converter were installed in the laboratory. The created automated SC parameter measurement equipment included SCs integrated into lamps, a data collector, a computer (PC), and software.

An analogue signal was converted into a digital one by an ADC-16 converter. The data collector was connected to the computer, enabling to register and collect data on the solar cell EMF. The PicoLog and Microsoft Excel programm packages were used for data collection and the programm packege Mathematica 8.0 for analysis. SCs parameters were measured continuously by recording the average 10 -minute values in the computer database.

Two polycrystalline silicon SCs with different photosensing areas of $5.58 \mathrm{~cm}^{2}$ and $10.08 \mathrm{~cm}^{2}$ for $1 \mathrm{SC}$ and $2 \mathrm{SC}$, respectively, were used in our experiments.

The $30 \mathrm{k} \Omega$ external resistances were connected to the polycrystalline solar cells to measure a drop of voltage in the resistances, i.e. the power of the electromotive force (Fig. 1). Internal resistances of the solar cells $(10 \Omega)$ were significantly lower than their external resistances.

The illuminance data, obtained from a company involved in the examination of solar modules, were used for the analysis of results. The illuminance was measured by a SunnySensorBox meteorological station mounted on a horizontal surface of the SC module. During our experiment, polycrystalline silicon solar cells integrated into lamps were also exhibited in the horizontal position.

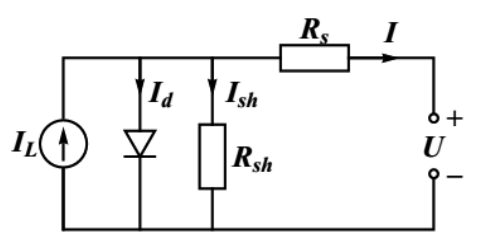

Fig. 1. Wiring scheme of solar cell.

Notations in the wiring scheme in Fig. 1 coincide with notations in Eq. (1).

\section{Electromotive force dependence on illuminance}

The SC monitoring data analysis revealed the existence of EMF periodic changes in the course of the day. Figure 2 presents EMF data of the total period of the experiment, grouped by hours and sun radiation activity $E$. The research results show that the EMF of the SC is observed from 5-6 a.m. when the sun goes up, and it is no longer recorded after sunset (9-10 p.m.). The highest values of EMF are recorded at $2-3$ p.m. when the sun is in the zenith. The total number of measurements equals to 780 .

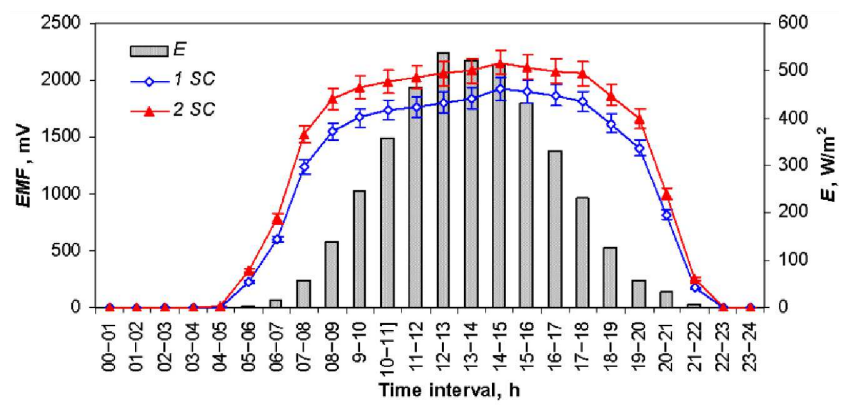

Fig. 2. Changes of SC EMF and illuminance $E$ during a day (March 8 - May 13, 2014). 
With sufficiently large amount of experimental data collected, the abundance of the data almost surely reflects the shape of the approximation curve, exactly like in our case. Figure 3 presents experimental data obtained when researching EMF dependence on illuminance: $\mathcal{E}=\mathcal{E}(E)$.

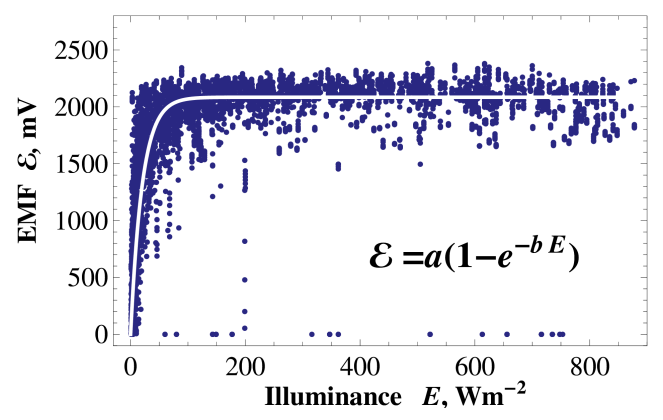

Fig. 3. Experimental data of EMF dependence on illuminance and the trend approximating it $(a=2085 \mathrm{mV}$, $\left.b=4.90 \cdot 10^{-2} \mathrm{~m}^{2} / \mathrm{W}\right)$.

There are two important features of Fig. 3:

a) firstly, the data start at the beginning of the coordinates. This confirms the physical nature of the dependence: no illuminance - no EMF;

b) secondly, an almost horizontal positioning of the data is clearly seen, which testifies the saturation mode of the $\mathcal{E}=\mathcal{E}(E)$ dependence: when illuminance $E$ reaches the value $E=100 \mathrm{~W} / \mathrm{m}^{2}$, the EMF no longer increases and equals about $2 \mathrm{~V}$.

Generally, the selection of the approximation curve is a rather complicated issue requiring a special grounding. In our case, we will show the experimental data presented in Fig. 3 as being of continuous dependence $\mathcal{E}(E)$ of EMF on illuminance $E$ (trend). We will consider this function not only as continuous, but as differentiated as well: $\mathcal{E}(E) \in C^{1}[a, b][23,24]$. To determine the trend function, we will make the presumption that the experimental data present the function of a fading exponent with respect to the saturation value

$$
\mathcal{E}=a\left(1-e^{-b E}\right) .
$$

In this dependence, $a$ and $b$ are unknown parameters to be determined. We obtain that $a=2085 \mathrm{mV}$ and $b=4.90 \cdot 10^{-2} \mathrm{~m}^{2} / \mathrm{W}$. Figure 3 presents experimental data and the trend approximating them (Eq. 2), with the parameters $a$ and $b$.

The physical meaning of parameters $a$ and $b$ is seen in Fig. 4. Parameter $a$ means the maximum asymptotic EMF value $\mathcal{E}_{\text {max }}$, and parameter $b$ is related to the illuminance $E$ measurement units enabling to move to nondimensional variables. The value $E_{0} \equiv 1 / b=20.4 \mathrm{~W} / \mathrm{m}^{2}$ is the value of illuminance at which EMF is reduced e times against the maximum value $\mathcal{E}_{\max }$ (Fig. 4).

The selection of the trend function is related to a physical law under study. When the physical law is known, the trend function is most often related to the law or its modification. There are cases, however, when it is difficult, or even impossible, to establish the trend function in

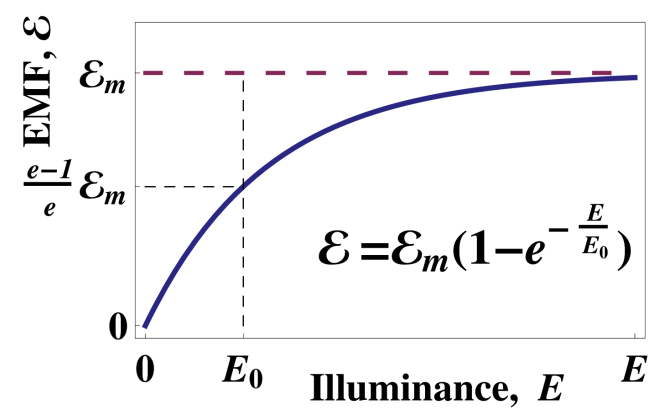

Fig. 4. Qualitative interpretation of the trend dependence $\left(\mathcal{E}_{\max }=2085 \mathrm{mV} ; E_{0}=20.4 \mathrm{~W} / \mathrm{m}^{2} ; \quad(\mathrm{e}-\right.$ 1) $\left.\mathcal{E}_{\max } / \mathrm{e}=1318 \mathrm{mV}\right)$.

advance. In such cases, several approximations with various mathematical functions have to be carried out, and the Pearson correlation coefficient should be used [25]. In this case, the correlation coefficient is the criterion which helps to select the best approximation curve.

The family of the current-voltage characteristics of a $\mathrm{Si}$ photoelement at various intensities of radiation were studied at the beginnings of solar energetics, see, e.g., [26, 27].

In addition to the experimental function (2), approximations with the power function and the logarithm function were performed. The results of these approximations are presented in Table. The crucial role in this table belongs to the Pearson correlation coefficient $R^{2}\left(0<R^{2}<\right.$ 1 ), and the closer $R^{2}$ is to unity the better.

TABLE

Various approximation functions, their parameters and correlation coefficients

\begin{tabular}{l|c|c|c}
\hline \hline Function & $a$ & $b$ & $R^{2}$ \\
\hline$a x^{b}$ & 953 & 0.1248 & 0.569 \\
$a \ln x+b$ & 276.9 & 225.7 & 0.727 \\
$a\left(1-\mathrm{e}^{-b x}\right)$ & 2085 & $4.90 \times 10^{-2}$ & 0.983
\end{tabular}

As we can see, when considering the value of the correlation coefficient, the approximation carried out with an exponential function is the best.

\section{Fluctuations of values as a noise $1 / f^{\alpha}$}

Let us consider the deviation of the EMF values from the trend described by the expression (2).

For a quantitative assessment of the obtained approximation and the errors while extrapolating formula (2) into an unknown region, we shall find the deviations of the obtained function from the real value of the EMF. We shall consider the deviation $\delta \mathcal{E}_{n}$ as a difference

$$
\delta \mathcal{E}_{n}=\mathcal{E}_{n}-\mathcal{E}(n), \quad n=1,2, \ldots
$$

where $n$ parameterizes the real values of the illuminance $E$. 
The dependence $\delta \mathcal{E}_{n}$ of real deviations could be analyze quantitatively. The mean square deviation (standard deviation) $\delta \mathcal{E}=256.009 \mathrm{~V} \approx 256.0 \mathrm{~V}$ from the mean value $\langle\delta \mathcal{E}\rangle=-53.161 \mathrm{~V}$. The most of the deviation value fit within the interval of two mean square deviations.

Like the initial distribution $\mathcal{E}_{n}$ of EMF values, the deviation dependence $\delta \mathcal{E}_{n}$ does not look very regular. The dependence is neither monotonous nor periodic. In case some periodicity is present in this dependence, it should be manifested in its Fourier spectrum. Figure 5 shows the $\left|a_{\max }\right|$ spectrum of dependence $\delta \mathcal{E}_{n}$, where

$$
a_{\max }=\frac{1}{\sqrt{n}} \sum_{j=1}^{n x_{j}} \delta \mathcal{E}_{j} \mathrm{e}^{\mathrm{i} 2(m-1) \pi x_{j}}, \quad x_{j}=\frac{j-1}{n} .
$$

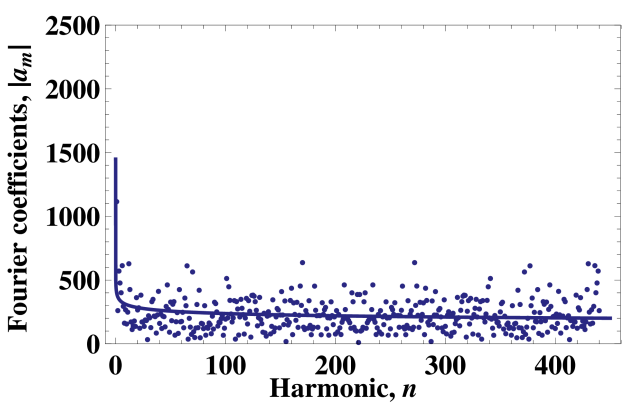

Fig. 5. The Fourier spectrum of the deviations $\delta \mathcal{E}_{n}$.

We see that the deviation dependence $\delta \mathcal{E}_{n}$ is not periodic, indeed, but similar to a noise with the spectral density function $S(f) \propto 1 / f^{\alpha}$.

To verify this hypothesis, we have to fit the Fourier spectrum. As follows from fitting, with a high accuracy, dependence $\left|a_{\max }\right|$ may be approximated by a function

$$
\left|a_{\max }\right| \approx \frac{396.731}{f^{0.1116}} \text {. }
$$

The spectral density function $S(f) \propto\left|a_{\max }\right|^{2}$, so we see that our hypothesis has come true: the EMF deviation dependence $\delta \mathcal{E}_{n}$ may be considered as noise $S(f) \propto$ $1 / f^{0.22}$.

This type of deviations, in the authors' opinion, is not incidental. The noise $1 / f$ for $\alpha=1$ is known to manifest itself in various branches of sciences such as physics, biology, finances. Although at present the nature of this noise is not clear, the universal character of the phenomenon is related to the properties of fractal multitudes [28]. However, in our case, the function $S(f) \propto 1 / f^{0.22}$ is close to white noise spectral density function, indicating that both the deviations and the initial distribution of the EMF are weakly fractal multitudes.

\section{The mathematical model as a dynamical system}

SC elements and modules represent a wide field for the mathematical modelling [23-25] of their properties. For instance, the SPICE modelling tool is typically used in the development of electrical and electronic circuits [20, 29]. An enhancement of a generic battery model, achieving a dynamic battery model for photovoltaic applications, was considered in [30]. Our efforts relate to the concept of a dynamical system.

According to the general definition, a dynamical system is a set of the $n$-dimensional smooth manifold $\mathcal{M}^{n}$ and a one-parameter group of diffeomorphisms $g^{t}$ (see e.g. [31])

$$
D S=\left\{\mathcal{M}^{n}, g^{t}\right\} .
$$

Is it possible to consider solution (2) as an evolutionary function of a dynamical system?

The first step: we have to consider the illuminance $E$ as a time variable. The next step: we have to find the differential equation and initial conditions corresponding to the function (2).

A trend is not yet a mathematical model. To find a differential equation the solution of which is the function (2), we will use the method of undetermined coefficients [32]. We will establish the function (2) derivative

$$
\mathcal{E}^{\prime}=a b \mathrm{e}^{-b E} \text {. }
$$

The concept of the dynamical system does not depend on the nature of the evolution parameter $t$. According to definition (6), the main aspects are the $n$-dimensional smooth manifold $\mathcal{M}^{n}$ and a one-parameter group of diffeomorphisms $g^{t}$. The parameter $t$ denotes time only according to the historical tradition.

Since there are two unknown coefficients in the function (2), a system of two equations, Eq. (2) and Eq. (7), is fully sufficient to establish the unknown differential equation of the mathematical model.

Let us write down of Eq. (2) and Eq. (7)

$$
\mathcal{E}=a\left(1-\mathrm{e}^{-b E}\right), \quad \mathcal{E}^{\prime}=a b \mathrm{e}^{-b E},
$$

where $\mathcal{E}^{\prime}$ means a derivative with respect to the illuminance $E$.

We will solve Eqs. (8) with respect to functions $\mathcal{E}$ and $\mathcal{E}^{\prime}$ by eliminating an independent variable - illuminance $E$. It follows from the first equation that

$$
a \mathrm{e}^{-b E}=a-\mathcal{E} \text {. }
$$

Upon entering the expression into the second equation, we obtain a differential equation

$$
\mathcal{E}^{\prime}=b(a-\mathcal{E}) \text {. }
$$

Considering the feature of the experimental data and the trend (2)

$$
\mathcal{E}(0)=0,
$$

we will get an answer to the modelling question: the differential Eq. (10), together with the initial conditions (11), make a Cauchy problem of the dependence under study.

Now let us make sure that the function (2) satisfies the differential equation (10) and the initial condition (11). Generally, a relevant programme package is suggested to be used for reaching this aim, but in our case the procedure is not complicated and can be performed manually: by entering function (2) into Eq. (10) and conditions (11), we obtain an identity. 


\section{Physical interpretation of the model}

Thus, we have not only the approximation of experimental measurements presented in Sect. 4, but also a mathematical model of the distribution of the experimental values described in Sect. 6. The natural question arises: how this mathematical model relates to the characteristic equation of the solar cell?

To determine the theoretical effect of the EMF $\mathcal{E}$ on the illuminance $E$, let us remind one of the basic properties of the photoeffect: at constant spectral composition of electromagnetic radiation the incidence on the photocathode saturation of the photocurrent is proportional to the irradiance of the cathode. In other words, the number of photoelectrons emitted from the cathode per one second is directly proportional to the illuminance $E: n \sim E$ or $I_{L}=b E$. Since the photocurrent is proportional to the number of photoelectrons, from the basic characteristic Eq. (1) we obtain the expression

$$
\frac{b}{I_{0}} E=\exp \left[\frac{q\left(U+I R_{\mathrm{s}}\right)}{\alpha k T}\right]+\frac{q\left(U+I R_{\mathrm{s}}\right)}{R_{\mathrm{sh}}}+\frac{I}{I_{0}}-1
$$

The EMF $\mathcal{E}$ corresponds to the voltage on the SC contacts or voltage $U$ in the case $R_{\mathrm{s}}=0$ (see the scheme in Fig. 1). As follows from Eq. (12), the dependence of EMF $\mathcal{E}$ on illuminance $E(\mathcal{E}=\mathcal{E}(E))$ has a transcendental character, and the inverse function $E=E(\mathcal{E})$ shows an exponential growth

$$
\frac{b}{I_{0}} E=\exp \left(\frac{q \mathcal{E}}{\alpha k T}\right)+\frac{q \mathcal{E}}{R_{\mathrm{sh}}}+\frac{I}{I_{0}}-1 .
$$

Just as in the case of the characteristic Eq. (2), Eq. (13) has no analytical solution and has to be solved numerically. As follows from the Sect. 4, the proposed function (2) is quite a good approximation of the theoretical dependence (13).

\section{Conclusions}

The modelling of the solar cell EMF and illuminance has shown that in case of $100 \mathrm{~W} / \mathrm{m}^{2}$ illuminance EMF reaches a value of around $2 \mathrm{~V}$ and then changes insignificantly with an increase of illuminance; consequently, this means that in case of the above illuminance the SC operates at its highest efficiency. The research results show that the illuminance reaches the value of $100 \mathrm{~W} / \mathrm{m}^{2}$ at 8-9 a.m., and this value decreases to $<100 \mathrm{~W} / \mathrm{m}^{2}$ at 7-8 p.m., i.e. SCs work most efficiently during the above hours.

The established differential Eq. (10), together with the initial condition (11), can be interpreted as a continuous dynamic system in which the role of time is played by illuminance $E$. This dynamic system is linear. Its phase portrait, with a change of the parameter $b$, includes straight lines of various trends, going through the beginning of the coordinates.

It should be stressed that in cases allowing a change of the other parameters of SC, which were fixed in our case, the determined dependency of SC EMF on illuminance
$\mathcal{E}=f(E)$ (2) can be more complicated. The corresponding differential equation can be a nonlinear one. As a consequence, the phase portrait of such a system will be different. However, there remains the main idea that the dependence of the crystal semiconductor (SC) EMF on illuminance can be interpreted as a dynamic system.

The general understanding that a dynamic system is a set of the $n$-dimensional smooth manifold $\mathcal{M}^{n}$ and a one-parameter group of diffeomorphisms (7) can be generalized. It is enough to require one-side differentiability instead of smoothness, whereas the group of diffeomorphisms can be restricted to its semigroup $g^{t+}$. The DS generalized in this way enables the usage of singular integro-differential operators instead of a classical evolution operator [33].

In our case, the proposed mathematical model is linear. The most fruitful results could be expected in the case of nonlinear mathematical models when the powerful methods of dynamical systems are used.

We see that the interpretation of the SC illuminance $E$ as the "time" variable is productive since it allows applying powerful DS methods for the SC mathematical modelling. Especially powerful this method should be in case of nonlinear and complicated behavior of the analyzed system. The other possible sphere of mathematical modelling is related to the analysis of the fluctuation of experimental values with respect to the trend.

\section{Acknowledgments}

One of authors A.J. acknowledges a support of the project code VP1-3.1-SMM-08-K-01-009 by the National Program of the Lithuanian Ministry of Education and Science in collaboration with the European Social Fund Agency.

\section{References}

[1] R.P. Mukund, Wind and solar power systems: design, analysis, and operation, CRC Press, Taylor \&Francis Group, Singapore 2006.

[2] T. Ma, Y. Nakamori, Energy 34, 873 (2009).

[3] T. Markvart, Photovolt. Bull. 1, 7 (2001).

[4] A. Luque, S.Hegedus, Handbook of Photovoltaic Science and Engineering, Wiley, New York 2011.

[5] V. Adomavičius, in: Official Journal of the Lithuanian Applied Sciences Academy, University of Klaipèda), Klaipèda 2010 (in Lithuanian).

[6] X.Wang, Z.M. Wang, High-Efficiency Solar Cells: Physics, Materials, and Devices, Springer, Berlin 2013.

[7] A. McEvoy, T. Markvart, L. Castaner, Practical Handbook of Photovoltaics, Academic Press, Oxford 2011.

[8] R. Palumbo, R.B. Diver, C.Larson, E.N. Coker, J.E. Miller, J. Guertin, J. Schoer, M. Meyer, N.P. Siegel, Chem. Eng. Sci. 84, 372 (2012).

[9] W. Sheline, L. Matthews, N. Lindeke, G.S. Duncan, R.D. Palumbo, Engineering Faculty Publications, 14 (2013). 
[10] R.B. Best, J.M.H. Aceves, J.M.S. Islas Manzini, I.F. Pilatowsky, R. Scoccia, M. Motta, Applied Thermal Eng. 50, 1447 (2013).

[11] N.L. Panwar, S.C. Kaushik, S. Kothari, Renew. Sustain. Energ. Rev. 15, 1513 (2011).

[12] P. Würfel, Physics of Solar Cells: From Basic Principles to Advanced Concepts, Wiley-VCH, Weinheim 2009.

[13] G.R. Timilsina, L. Kurdgelashvili, P.A. Narber, Renew. Sustain. Energ. Rev. 16, 449 (2012).

[14] M. Šúri, T.A. Hiild, E.G. Dunlop, PVGIS: a webbased solar radiation database for calculation of PV potential in Europe, Int. J. of Sustain. Energ. 24, 55 (2005).

[15] V. Mačiulis, Energijos erdve 5, 34 (2010) (in Lithuanian).

[16] National Energy Strategy, project adopted by the Seimas of Republic of Lithuania on October 6, 2010, Vilnius (in Lithuanain).

[17] J. Nelson, The Physics of Solar Cells, Imperial College Press, London 2003.

[18] S.J. Fonash, Solar cell device physics, Academic Press, Amsterdam 2010.

[19] E. Lorenzo, Solar Electricity: Engineering of Photovoltaic Systems, Progensa, Spain 1994.

[20] S. Silvestre, A. Boronat, A. Chouder, Appl. Energ. 86, 1632 (2009).

[21] A.R. Jha, Solar cell technology and applications, CRC Press. Taylor \&Francis Group LLC., Boca Raton 2010 .
[22] M. Richter, M. Ya. Amusia, S. V. Bobashev, T. Feigl, P. N. Juranic, M. Martins, A. A. Sorokin, K. Tiedtke, Phys. Rev. Lett., 102, 163002 (2009).

[23] R. Slingerland, L. Kump, Mathematical Modelling of Earth's Dynamical Systems, Princeton University Press, Princeton 2011.

[24] A.A. Samarskij, A.P. Mihajlov, Mathematical modeling: Ideas, Methods, Examples, 2 ed., Moscow 2001 (in Russian).

[25] W.P. Fox, F.R. Goirdano, M.D. Weir, First Course in Mathematical Modeling, Tomson Learning, Kindom 2003.

[26] P.A. Iles, IRE Trans. Milit. Electron. 6, 5 (1962).

[27] A. Ambroziak, Construction and technology of photovoltaic devices, Scientific-Technical Publishers, Warsaw 1965 (in Polish).

[28] B. Mandelbrot, The Fractal Geometry of Nature, W.H. Freeman, New York 1983.

[29] D. Guasch, S. Silvestre, Progr. Photovolt. Res. Appl. 11, 193 (2003)

[30] L. Castaner, S. Silvestre, Modelling photovoltaic systems using PSpice, John Wiley, Chichester 2002.

[31] V.I. Arnold, Ordinary differential equations, Springer Verlag, Berlin 1992.

[32] W.A. Adkins, M.G. Davidson, Ordinary differential equations, Springer, New York 2012.

[33] P. Miškinis, Nonlinear and nonlocal integrable models, Technika, Vilnius 2003 (in Lithuanian). 